\title{
Advancing surgical education
}

\author{
Dimitrios Stefanidis ${ }^{1}$
}

Published online: 22 February 2022

(c) The Author(s), under exclusive licence to Association for Surgical Education 2022

\section{Dear Reader:}

Welcome to the inaugural issue of Global Surgical Education the new journal of the Association for Surgical Education (ASE). The ASE was established in 1980 by Dr Royce Laycock along with a number of visionary surgeons who recognized the need to incorporate best practices in surgical education. Since that time, the society has grown considerably and currently has over 1200 members from various surgical disciplines including surgeons, surgical trainees, $\mathrm{PhD}$ educators, nurses, coordinators, medical students, and other learners. Since its inception, the society has been publishing select content from its annual meeting in the American Journal of Surgery, typically in its January or February issue.

So why a new journal now? The growth of the ASE and the education content of its annual meeting has outgrown the ability of the AJS to accept our content. Further, surgical education has seen unprecedented growth over the last three decades; a simple Pubmed search using the term "surgical education" produced 360 articles in 1981 while in 2021 this number had climbed to 15,350 , a 42 times increase! Clearly, the interest in surgical education research is increasing exponentially in recent years; however, authors submitting their education science-focused papers still experience challenges getting them accepted at biomedical journals as reviewers in those journals are often unfamiliar or have limited understanding of such work. Therefore, our intent is to provide a home for manuscripts that use high-quality education methodology and advance the science by attracting the best work in the field. Importantly, as evidenced by our journal's name, we want it to serve the surgeon educator globally and provide a venue that allows surgeons from around the world to exchange ideas, learn from each other, and advance surgical education science.

Dimitrios Stefanidis

dimstefa@iu.edu

1 Department of Surgery, Indiana University School of Medicine, Indianapolis, IN, USA
To that end, we have assembled an outstanding multidisciplinary and international Editorial Board with diverse representation from many different surgical disciplines and education domains and have chosen four qualified individuals to serve as Associate Editors. All Editorial Board members have been chosen for their expertise in different aspects of surgical education. Further, we have assembled a group of highly qualified peer-reviewers, who, along with the Editorial Board and editors, will be responsible for a publication that is rigorous in its peer-review, attentive to the unique challenges of research and scholarship in education, and dedicated to advancing the field. We promise that the review process for submitted manuscripts will be fair, unbiased, thorough, and timely. To that end and to maintain the integrity of the reviews, we chose our review process to be blinded; we are interested in the best ideas and work not where or from those ideas and work are coming from. Further, our journal includes a Diversity, Equity, and Inclusion (DEI) associate editor to ensure that we stay committed to DEI in the content we publish. We strive for a balance of innovative and scientifically sound articles that address the contemporary issues surgical educators face. We invite original research articles, reviews, opinion papers, and new concepts and commentaries on pertinent topics. Topics of interest include novel teaching methods and strategies, curriculum development, procedural and team simulation with an emphasis on education outcomes, faculty development, assessment tool development, use of artificial intelligence to improve performance assessment, surveys on important and pertinent educational topics, evidence-based reviews of current teaching practices, and any topic pertaining to the surgical learner and surgeon educator.

We look forward to making our new journal a success and hope that the readers equally look forward to reading its content avidly. I would be remiss if I did not recognize the strong support of our publisher, Springer, who saw our journal's potential and gave us the opportunity to create this online only journal focused solely on surgical education. We are indebted to Sverre Klemp and David Stanmore who worked tirelessly with us to create this journal. 
In the first published papers in our new journal, we are pleased to include a provocative paper by one of the past ASE presidents, Dr Gary Dunnington, who presents his views and suggestions for how to build a surgical education culture in your department, and by the immediate past president of the ASE, Dr Daniel Scott, who provides an outstanding overview of the society's history and strengths. In addition, you can find several interesting original papers on a variety of surgical education topics.

I would also like to emphasize that a new journal is a work in progress. We seek feedback from the Board and membership of Association for Surgical Education and the wider readership we aim to establish. We are open to your innovative ideas and encourage all of you to help us create the finest publication possible by sharing with us your thoughts on the direction of our new journal Global Surgical Education: Journal of the Association for Surgical Education. 